\title{
Evaluación interdisciplinaria estomatognática y postural en un paciente pediátrico respirador oral: Reporte de caso
}

\author{
Interdisciplinary stomatognathic and postural assessment in a pediatric mouth-breathing patient: A case report \\ Julialba Castellanos-Ruíz ${ }^{*}$ orcid.org/0000-0002-4857-7330 \\ Olga Patricia López-Soto'1 orcid.org/0000-0002-5045-820X \\ Lina María López-Soto ${ }^{1}$ orcid.org/0000-0002-9588-0420 \\ Yéssica Paola López-Echeverry ${ }^{1}$ orcid.org/0000-0002-2494-4255 \\ Montserrat de Alba Sánchez² orcid.org/0000-0002-8161-0243 \\ Lilian Ortega-Oviedo² orcid.org/0000-0002-1132-3919 \\ Mayary Stefani Ramírez-Cuellar1 orcid.org/0000-0001-7494-9372 \\ Sandra Milena Vélez-Arango' ${ }^{1}$ orcid.org/0000-0001-6224-6725 \\ Eola De la Rosa-Marrugo ${ }^{1}$ orcid.org/0000-0002-0719-3461
}

1. Departamento de Movimiento Humano. Universidad Autónoma de Manizales. Manizales, Colombia.

2. Benemérita Universidad Autónoma de Puebla. Zaragoza, México.

\section{Resumen}

Introducción: La respiración oral se asocia con trastornos de los órganos del habla, que generalmente se combina con deformidades faciales y problemas de oclusión dental. Objetivo: Evaluar interdisciplinariamente la fisiología del sistema estomatognático y postural en un paciente respirador oral de 11 años. Presentación del caso: Paciente de sexo masculino, se le realizó examen orofacial mediante mioescanografía, dinamometría y electromiografía, técnica de Payne y prueba Rosenthal. Se evaluó alineación postural, estabilidad con índice de Romberg y presiones plantares con baropodometría. Resultados: El paciente presentó mal oclusión clase I, hipoplasia maxilar hiperdivergente y micrognatismo, en actividad muscular, índice de asimetría 34\% en maseteros durante la masticación, en temporales durante la protrusión la simetría fue de $67 \%$ y de $62 \%$, alteración de la estabilidad estática con Índice de Romberg de 1,22 y distribución de presión plantar en pie izquierdo 42,2\% y derecho 57,8\%. Conclusiones: El sujeto presentó disminución en actividad de fuerza muscular orofacial, alteraciones en la alineación de cabeza, hombros, región pélvica, alteración de la estabilidad estática y presiones plantares con mayor distribución en retropié bilateral y miembro inferior derecho.

Palabras clave: Postura; respiración bucal; sistema estomatognático; niños. (Fuente: DeCS, Bireme).

\begin{abstract}
Introduction: Oral respiration is associated with speech organs disorders, which are generally combined with facial deformities and dental occlusion problems. Objective: To conduct an interdisciplinary assessment of the physiology of the stomatognathic and postural system in a 11-year old mouth-breathing patient. Case presentation: The male subject underwent orofacial examination using myoscanography, dynamometry, electromyography, Payne technique, and Rosenthal test. Postural alignment, stability (Romberg index) and plantar pressures (baropodometry) were evaluated. Results: The subject displayed: class I malocclusion; hyperdivergent maxillary hypoplasia, and micrognathism in muscular activity; a $34 \%$ asymmetry index in masseters during mastication; the symmetry in temporals was $67 \%$ and $62 \%$ during protrusion; alteration of the static stability with a Romberg index of 1.22 ; and $42.2 \%$ and $57.8 \%$ plantar pressure distributions in the left and right feet, respectively. Conclusions: The subject showed a reduction in orofacial muscle strength activity, alterations in the alignment of the head, shoulders and pelvic region, an alteration of the static stability, and plantar pressures with a greater distribution on the bilateral hindfoot and right lower limb.
\end{abstract}

Keywords: Posture; mouth breathing; stomatognathic system; children. (Source: DeCS, Bireme). 


\section{Introducción}

La respiración oral (RO) es una condición clínica que se caracteriza por una respiración suplementaria mixta o bucal, que reemplaza un patrón de respiración exclusivamente nasal ${ }^{(1)}$. Se asocia con trastornos de los órganos del habla y generalmente se combina con deformidades faciales; con el potencial de progresar a enfermedades cardiorespiratorias, endocrinas y trastornos del sueño, que pueden comprometer el comportamiento y el rendimiento personal ${ }^{(2)}$.

Clínicamente, la RO se caracteriza por un aumento en la dimensión del tercio inferior de la cara, paladar profundo y estrecho, maloclusión clase II, mordida cruzada posterior y abierta anterior, labio superior corto e inferior evertido, retrusión mandibular, incremento en la lordosis cervical $\mathrm{y}^{(3)}$ cabeza en posición adelantada(4). Se ha reportado que la respiración oral causa un crecimiento facial anormal(5), problemas de atención asociados con trastornos del sueño y una reducción en la calidad de vida(6). Además, se ha informado una mayor carga de oxígeno en la corteza prefrontal al cambiar de la respiración nasal a la respiración bucal(7). De esta manera, en el campo de la odontología se ha investigado principalmente las características de la respiración bucal, con el propósito de descubrir sus efectos, identificar a pacientes respiradores bucales habituales, así como sus particularidades oclusales $(8,9)$.

Los factores etiológicos de la RO pueden ser obstrucciones como hipertrofia palatina y faríngea y la desviación del septum nasal(10,11). La RO es funcional cuando resulta de hábitos orales de larga duración, alteraciones musculares, edema transitorio de la mucosa nasal como la rinitis intermitente y la obstrucción de vías aéreas reparadas(12,13). Las consecuencias de la RO incluyen cambios craneofaciales y corporales $^{(14)}$ como infecciones y alergias, alteraciones musculares e inflamación transitoria de la mucosa nasal ${ }^{(15,16) .}$

La evaluación visual se usa con frecuencia $(97,2 \%)$ para identificar los hallazgos característicos de la respiración bucal(10,17) en esta, se observa en el participante la presencia de facies adenoideas, hipertrofia de amígdalas palatinas y tabique nasal desviado(18); y se determina si la ruta de la respiración bucal está cerrada en reposo ${ }^{(19)}$.
El diagnóstico de la RO es predominantemente clínico, dado que es una condición que incluye varios sistemas orgánicos. Algunos exámenes y pruebas pueden ser complementarios, al identificar sus factores etiológicos(2), éstos pueden ser concomitantes, al actuar de forma intermitente o continua, además de la complejidad de cuantificar su magnitud y severidad. La RO al convertirse en una acción repetitiva rompe el equilibrio del sistema estomatognático y puede generar maloclusiones como mordidas cruzadas, mordidas abiertas y colapso de paladar. En general, el diagnóstico y tratamiento de las personas con RO se realiza en forma independiente por cada especialista, olvidando generalmente los efectos en la postura corporal de esta clase de respiración. Se requiere entonces un abordaje interdisciplinario tanto desde los procesos de evaluación como de intervención, ya que los cambios generados en el sistema musculoesquelético y en la capacidad respiratoria, conducen a un uso inadecuado del patrón respiratorio y posturas compensatorias que exacerban esta condición de salud(20).

El objetivo de este reporte de caso es evaluar interdisciplinariamente la fisiología del sistema estomatognático y postural en un sujeto respirador oral de 11 incluyendo en la evaluación postural la estabilidad estática, límites de estabilidad y presiones plantares. Estudio que se realizó con participación interdisciplinaria de la odontología, la fisioterapia, la fonoaudiología y la ingeniería biomédica, en un proceso de evaluación conjunta, a través de la aplicación de pruebas clínicas con el uso de tecnología.

\section{Presentación del caso}

Estudio de caso de un sujeto masculino de 11 años de edad, remitido del servicio del posgrado de ortodoncia por posible alteración postural. El participante registraba antecedentes médicos de respiración oral desde los 9 años; rinitis y alergia al polvo desde los dos años y estrabismo. En el momento del examen no estaba medicado. Se observó una relación molar de Angle Clase I bilateral, canina Clase II, over jet de $1 \mathrm{~mm}$, over bite del $25 \%$, línea media inferior desviada a la izquierda, perfil recto y proquelia, como se observa en la Figura 1. 


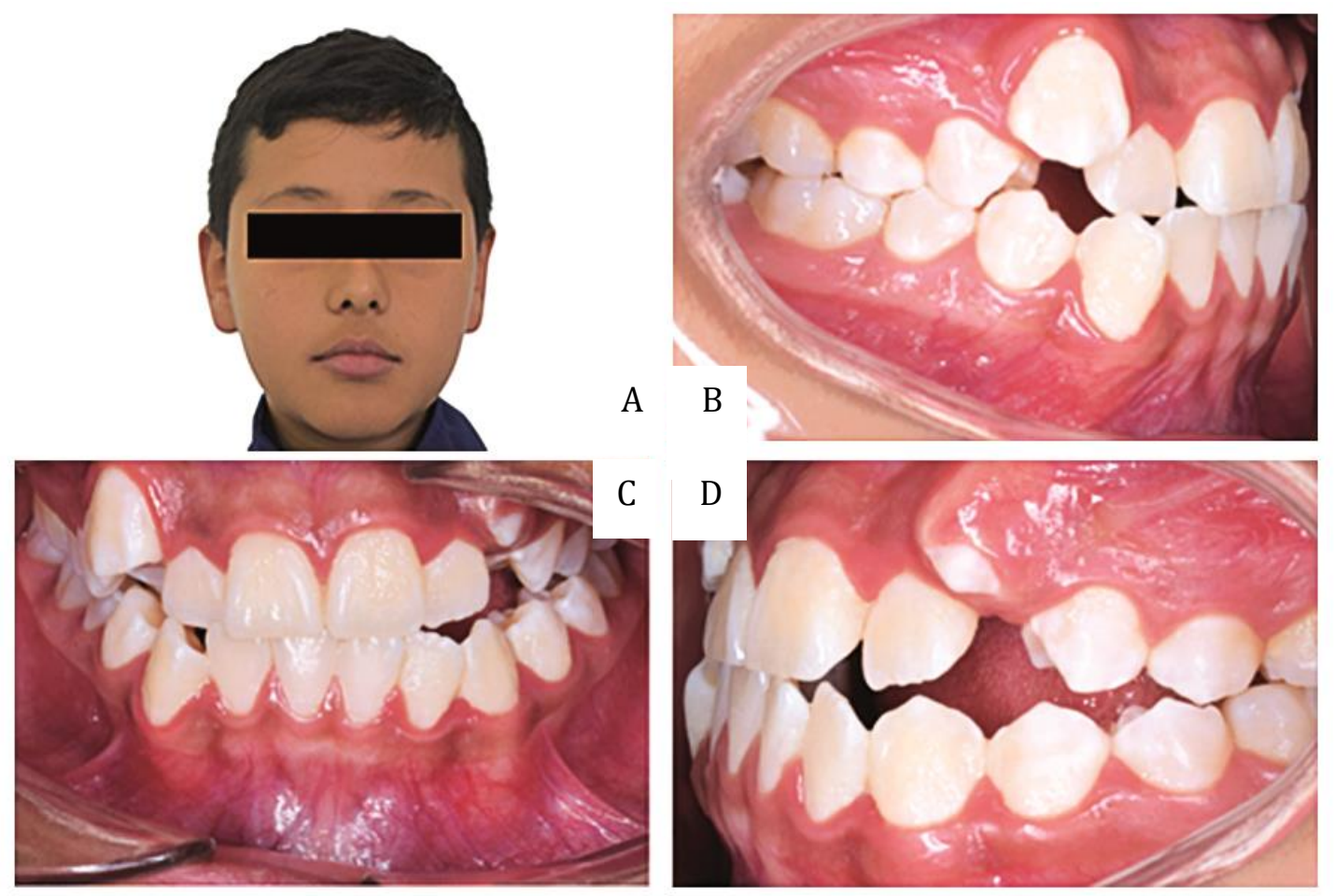

Figura 1. Fotografías clínicas del sujeto y su condición oclusal. A: Paciente hiper-divergente, con línea facial no coincidente, presenta tercio inferior facial aumentado. B: Relación molar clase I derecha, relación canina clase II, overjet disminuido, se observa mordida cruzada entre 12/42-43, erupción ectópica 13. C: Relación molar clase I izquierda, la relación canina no aplica ya que el diente 23 está en erupción (ectópica), mordida cruzada entre 25/35 se observa overjet y overbite disminuido. D: Dentición permanente, erupción ectópica de 13 y 23, presenta micrognatismo transversal en maxilar superior, apiñamiento superior e inferior, overbite disminuido se evidencia línea media dental inferior desviada a la izquierda.

El usuario fue diagnosticado en tres sesiones por un equipo interdisciplinario compuesto por un residente de ortodoncia, una fisioterapeuta y una fonoaudióloga terapista miofuncional. Un ingeniero biomédico registró y organizó las señales electromiográficas y estabilométricas. La anamnesis del participante fue reportada por sus padres. Las observaciones de las pruebas diagnósticas siguieron los protocolos del laboratorio de postura de la entidad universitaria.

Con el fin de realizar un diagnóstico integral del participante se decidió aplicar, además del examen rutinario de postura, una prueba de estabilometría y una de podometría. Asimismo, se aplicaron pruebas para determinar la fuerza y la actividad orofacial durante la función masticatoria y el reposo utilizando los equipos Myoescaner y Dinamómetro considerando que es importante confirmar si estos músculos están alterados como es usual en estos

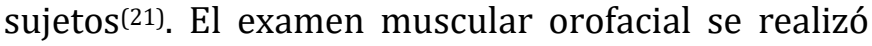

mediante Mioescanografía, equipo que permite determinar la fuerza de los músculos maseteros, temporales y lingual en función, que midió la tensión fisiológica de los músculos censados por medio de un plug, que permitió medir la fuerza de compresión labial, fuerza de proyección lingual y contracción de los maseteros. La mioescanografía se realizó utilizando un equipo Pounds Myoescanner, eilcoTechnologyinc, D-926119T8 Hoxt, 1volt=F.S, pt 2500. La fuerza compresiva de los orbiculares se midió por dinamometría con un equipo Marca Chatillon, N.Y, U.S.A., model CHA IN-10. Se utilizó un muelle de acero que termina en un gancho dentro de un tubo cilíndrico graduado; este gancho estaba unido a un cordón de seis pulgadas, que finalizaba en un botón de una pulgada de diámetro. El botón se ubicó en la parte anterior de los incisivos y posterior de los labios, el investigador haló el dinamómetro hasta que el botón fue expulsado y después se leyó el resultado. 
Para el registro de la actividad eléctrica de los músculos orofaciales (sEMG) se realizó una electromiografía de superficie(22), para lo cual se utilizó un equipo Biometrics DataLog MWX8 ${ }^{\circledR}$. El electromiógrafo detectó el potencial eléctrico generado por las células musculares, cuando estas células se activaron neurológicamente al masticar. Los electrodos se posicionaron de la siguiente manera: para los maseteros y temporales: se solicitó al sujeto realizar un cierre dentario con máxima fuerza, se palpó el músculo masetero y el músculo temporal, un electrodo activo se ubicó en el punto motor y un electrodo de referencia fue ubicado $1 \mathrm{~cm}$ por debajo (Figura 2).

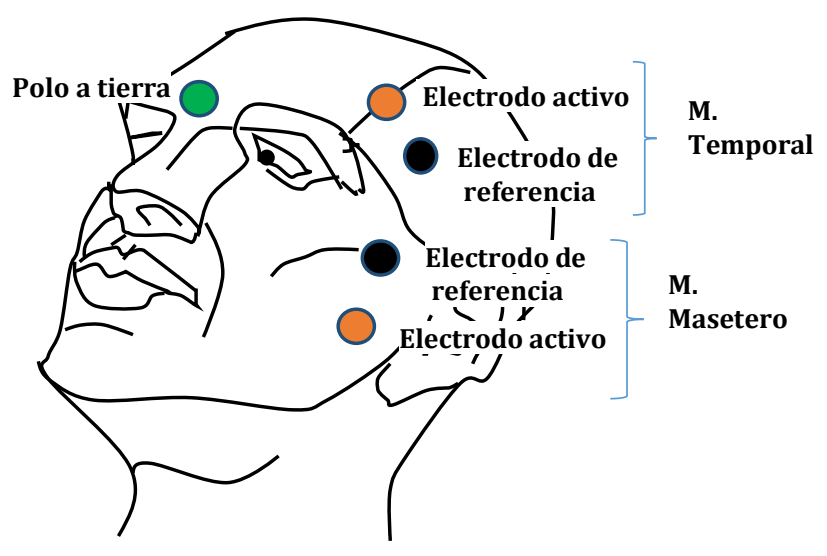

Figura 2. Posicionamiento de los electrodos activos y de referencia en los músculos masetero y temporal.

Para la valoración de las funciones del sistema estomatognático, se siguieron los protocolos preestablecidos para la aplicación de las pruebas fonoaudiológicas. La succión se valoró mediante preguntas por los hábitos orales aplicando el cuestionario utilizado por el estudio de SilvestriniBiavati del 2016(23), que indaga por los hábitos orales relacionados con la succión (succión digital durante el día y la noche, y si había succión con cuál dedo) que fue respondido oralmente por los padres y complementado con observación de los dedos por parte del investigador (forma y coloración como signo de hábito). La deglución se valoró mediante la Técnica de Payne que consiste en la detección de los puntos de contacto de la lengua en el paladar y la cara interior de los dientes durante la deglución. Esta prueba utiliza fluoresceína para marcar el contacto de la lengua al deglutir, que se observa por contraste con una lámpara de luz negra.
En la respiración se valoró la permeabilidad nasal y la capacidad respiratoria utilizando la prueba de Rosenthal, que consideró 20 actos respiratorios, primero con las 2 narinas, luego con cada una de ellas, si el sujeto es respirador bucal, abrirá la boca antes de finalizar la prueba. La fono-articulación se valoró con el test de articulación dónde se solicitó al usuario repetir palabras con los fonemas $/ S /, / r /$ y $/ \mathrm{rr} /$, en posición inicial, media y final en la palabra, para determinar si presentaba rotacismo y sigmatismo, alteraciones relacionadas con la maloclusión que podrían presentar los respiradores orales(24).

La evaluación de la alineación postural se realizó en vista posterior, anterior y lateral. Se empleó la plataforma de baropodometría, para el análisis y registro de la posición y los desplazamientos del centro de gravedad del sujeto en posición estática en el interior de su polígono de sustentación, así como el reparto en $\mathrm{Kg}$. del peso total en cada miembro inferior y las incidencias de las entradas sensoriales de los mecanorreceptores posturales (ojo, boca, oído), en las disfunciones de postura, mediante el programa informático G-Study, marca TS, modelo PWalk(25).

En la misma plataforma se evaluó la estabilidad estática, por medio del Índice de Romberg con ojos abiertos y cerrados, que permite identificar y analizar las oscilaciones del centro de presión a nivel medio lateral y anteroposterior. También se realizó la prueba de límites de estabilidad, para evaluar el control voluntario en el desplazamiento de su centro de gravedad, hacia sus límites máximos de estabilidad(26), con la misma plataforma de Baropodometría descrita anteriormente, con desplazamiento de su centro de gravedad en sentido antero-posterior y medio lateral con ojos abiertos y con ojos cerrados(27).

\section{Consideraciones éticas}

Para evaluar al usuario, un acudiente firmó el consentimiento informado, aprobado por el Comité de Bioética de una entidad universitaria (Acta No 081 del 2018). De acuerdo a la Resolución 8430 de 1993 fue considerada una investigación con "Riesgo mayor al mínimo” por el usuario ser menor edad. 


\section{Resultados}

En la dinámica mandibular se obtuvieron rangos de movimiento en milímetros (mm), para apertura de 44 , en lateralidad derecha de 11, lateralidad izquierda de 5 y protrusión de 8,5, no presentó signos de deflexión, desviación o ruido. Al examen odontológico hubo relación maxilar hiperdivergente y micrognatismo transversal, mal posición dental, gingivitis, apiñamiento superior e inferior y mordida cruzada posterior. Se presentó un imbalance muscular orofacial con disminución en las fuerzas compresivas de los músculos orbicular $(0,2 \mathrm{lb})$, maseteros (Der: 0,2 lb Izq: 0,1 lb) y lingual $(0,6 \mathrm{lb})$.

En la deglución hubo ausencia de contracción de temporales (anormal), músculo orbicular, buccinador y mentón activos (anormal). Se presentó dislalia con distorsión del fonema /r/. La actividad muscular fue valorada según la cualificación de la intensidad de la actividad muscular propuesta por Basmajian(28). La actividad de maseteros, temporales y buccinador fue moderada y la del orbicular de los labios marcada (Tabla 1). El índice de asimetría se considera compatible con una función normal hasta un valor de $18 \%$ según Ferrario et al(29). El participante registró asimetría en maseteros y temporales durante la protrusión y durante la máxima intercuspidación (Tabla 2).

En la evaluación de la alineación postural, en la vista posterior, se observó cabeza con inclinación lateral derecha, hombro, ángulo inferior de la escápula izquierda y cresta ilíaca izquierda descendidos, pie normal izquierdo y tendencia a pie cavo en el derecho. En vista lateral, se visualizó cabeza y abdomen en posición adelantada, hiperlordosis lumbar, hombro en protracción, hiperlordosis lumbar, abdomen y cabeza en posición adelantada. En vista anterior, se reportó el lóbulo de la oreja derecha y el hombro del mismo lado descendidos, cresta ilíaca izquierda descendida y genu valgo.

A nivel de estabilometría, el Índice de Romberg fue de 1,22, indicando alteración en la estabilidad estática(30). En la distribución de las presiones plantares se observó mayor presión plantar en el pie derecho $(57,8 \%)$ vs pie izquierdo $(42,2 \%)$ y mayor presión a nivel de retropié bilateral como se observa en la Figura 3.

Para límites de estabilidad se presentó un incremento en la amplitud y frecuencia del balanceo. Con respecto a la tipología de pie, el participante presenta a nivel de miembro inferior pie izquierdo normal y arco de pie derecho ligeramente alto.

Tabla 1. Promedio en microvoltios de la actividad durante la masticación

\begin{tabular}{lc}
\hline & $\mathbf{X}$ \\
\hline Actividad masetero & \\
Derecho & 159,21 \\
Izquierdo & 187,77 \\
Actividad temporal & \\
Derecho & 265,73 \\
Izquierdo & 262,67 \\
Actividad orbicular & \\
Derecho & 367,71 \\
Izquierdo & 352,95 \\
Actividad buccinador & \\
Derecho & 155,72 \\
Izquierdo & 154,61 \\
\hline X: promedio en microvoltios \\
Insignificante: 0-50; ligera 51-150; Moderada 151-300; marcada 301- \\
500; mayor a 501 muy marcada.
\end{tabular}

Tabla 2. Porcentaje de asimetría muscular en función

\begin{tabular}{llr}
\hline \multicolumn{1}{c}{ Ejercicio } & \multicolumn{1}{c}{ Músculo } & Caso \% \\
\hline Masticación & Maseteros & -34 \\
& Temporales & -10 \\
& Buccinadores & -6 \\
& Orbiculares & - \\
Protrusión & Maseteros & -8 \\
& Temporales & -67 \\
& Buccinadores & -10 \\
& Orbiculares & 21. \\
Contracción voluntaria & Maseteros & -17 \\
en máxima & Temporales & 62 \\
intercuspidación & & \\
Indice de asimetría: Compatible con una función normal hasta un valor \\
de 18\%. Signo negativo $(-)$ & asimetría izquierda. Signo positivo (+) \\
asimetría derecha &
\end{tabular}




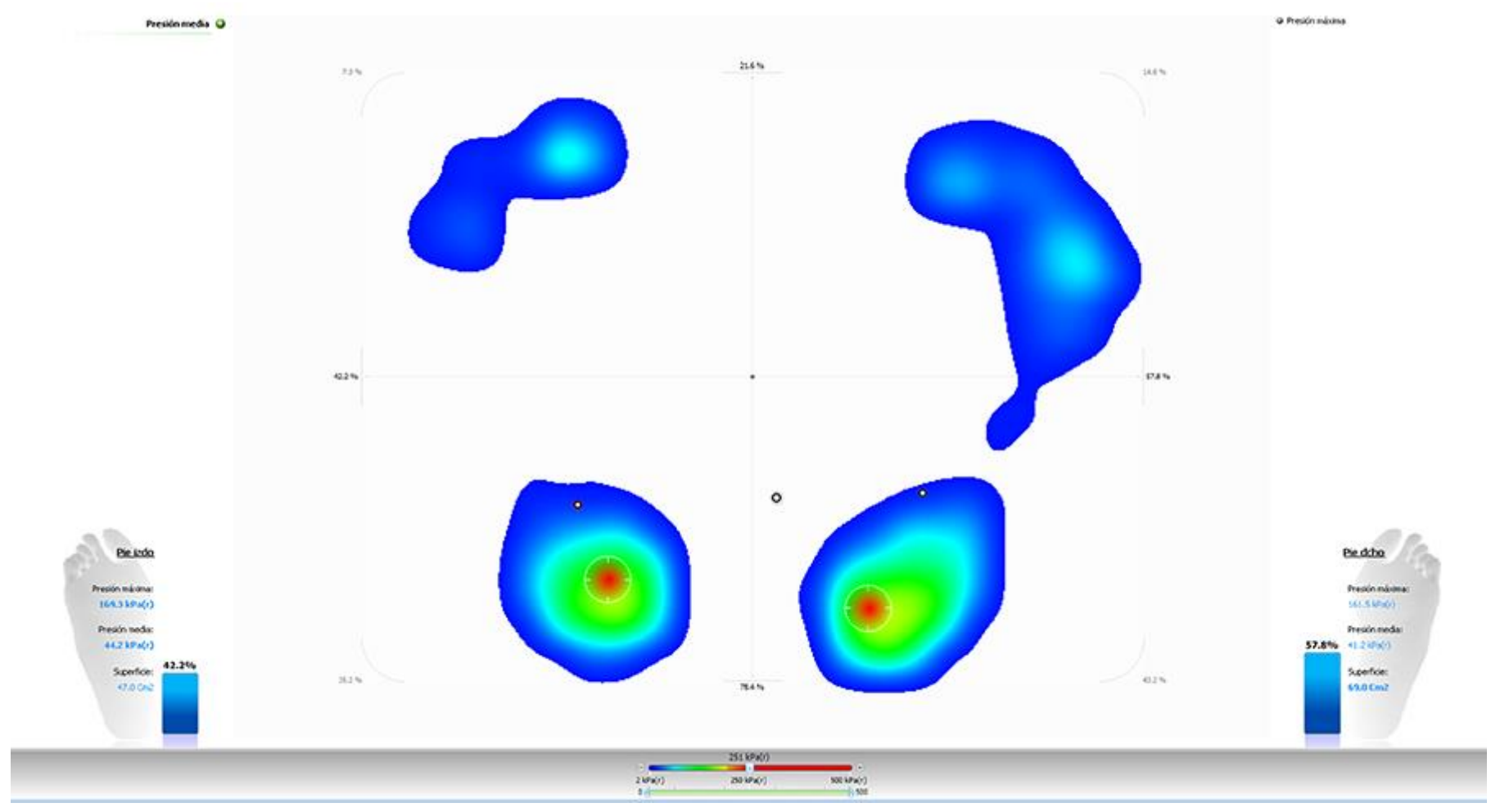

Figura 3. Distribución de presiones plantares. En la figura de la izquierda se observa dicha distribución por medio de una escala termográfica; el color azul indica, zonas de menor presión y con rojo, la zona de mayor presión; en este caso, la figura indica mayor presión en retropié, y la distribución de la presión plantar fue del 42,2\% en miembro inferior izquierdo. En la figura derecha, la mayor presión también se da en retropié, con mayor carga sobre el talón medial, la distribución de la presión plantar fue del 57,8\% en miembro inferior derecho.

\section{Discusión}

Este reporte de caso permitió la valoración interdisciplinaria de las funciones oro-faciales, dentales y posturales de un sujeto preadolescente con respiración bucal diagnosticada. Los músculos masticatorios maseteros y temporales presentaron asimetría durante la masticación y la máxima intercuspidación. La simetría en la actividad muscular se asocia con una fisiología adecuada. Ono et al.,(31) han sugerido que la RO afecta la actividad de los maseteros, la obstrucción de la vía aérea nasal disminuye la actividad de este músculo como efecto del sistema gamma aferente (motoneuronas gamma). Ikenaga et al(21). El individuo del presente estudio tuvo disminuida la fuerza de los músculos maseteros y orbiculares. Hsu y Yamaguchi en el 2012(33) concluyeron que la RO disminuye el grado y el número de ciclos masticatorios, lo que resulta en una disminución de la dimensión vertical en los dientes posteriores y con frecuencia en una mordida abierta.
En la evaluación de alineación postural, el usuario presentó posición adelantada de la cabeza. Okuro et al.,(20) afirman que la postura en personas con RO se modifica para facilitar la entrada del aire a través de la orofaringe. Cuccia et al.,(33) concluyeron que en la RO se crea un imbalance muscular y una mayor extensión de la cabeza en relación con la columna cervical. Otros autores plantean ${ }^{(34)}$ que en estos niños aumenta la tensión en los músculos de la cabeza y hombros, cambiando su posición en las direcciones anteroposterior o lateral. El participante presentó cabeza con tendencia a la inclinación lateral derecha, y en vista lateral, cabeza y abdomen en posición adelantada, esto coincide con el estudio realizado por Cuccia et al(33). El sujeto del estudio mostró alteración en la posición de escápula y hombros, Neiva et al.,(35) evidenciaron una elevación escapular en niños RO. Yi et al.,(36) reportaron lordosis cervical reducida, aumento de la cifosis torácica y lordosis lumbar aumentada, resultados al presente estudio. 
La presión plantar en el participante estuvo aumentada en el retropié, seguido del antepié y del medio pie; sin embargo, en el estudio realizado por Martínez-Nova et al.,(37) el pico máximo de presión se encontró en el antepié, seguido de retropié y mediopie. En el presente caso, a nivel estabilométrico se observa que la diferencia entre los picos de las oscilaciones anteroposteriores y mediolaterales fueron mayores con ojos abiertos y cerrados, mostrando dificultad en el usuario para mantener el equilibrio en posición estática, como lo explica Tafner(38), esto, asociado a un índice de Romberg mayor a uno.

\section{Conclusiones}

El sujeto respirador oral presentó disminución en actividad de fuerza muscular orofacial, alteraciones posturales y en la estabilidad estática. Los hallazgos del presente reporte de caso, no permiten proporcionar conclusiones definitivas por el tipo de estudio, no obstante, evidenció la posibilidad de que las alteraciones en el sistema estomatognático influyan en la alineación postural del sujeto, resaltando la necesidad de que este tipo de pacientes sean evaluados por un equipo multidisciplinario.

\section{Agradecimientos}

Al Laboratorio de Análisis de Movimiento de la Universidad Autónoma de Manizales por su colaboración, a Julián Marín, Juan Carlos Vinasco, Thomas Lock y Rafael Tostes del centro de Traducción de la U. Autónoma de Manizales por la traducción del resumen al inglés y al portugués.

Conflicto de intereses: Los autores declaran no tener conflicto de intereses.

\section{Referencias}

1. Barros J, Becker H, Pinto J. Evaluation of atopy among mouth-breathing pediatric patients referred for treatment to a tertiary care center. J Pediatr (Rio J). 2006;82(6):45864. Disponible en: https://doi.org/10.2223/jped.1561

2. Marques-Felcar J, Rafael-Bueno I, Silva-Massan AC, PereiraTorezan R, Rosa-Cardozo J. Prevalence of mouth breathing in children from an elementary school. Cien Saude Colet. 2010;15(2):427-35. Disponible https://doi.org/10.1590/s1413-81232010000200020

3. Korbmacher H, Eggers-Stroeder G, Koch L, Kahl-Nieke B. Correlation between anomalies of the dentition and pathologies of the locomotor system: a literature review. J Orofac Orthop. 2004;65(3):190-203. Disponible en: https://doi.org/10.1007/s00056-004-0305-3
4. Arthur J, NowaK J, Christensen T, Mabry J, Twsend M. Oral Habits. Pediatr Dent. 2019;386-93.

5. Chambi-Rocha A, Cabrera-Domínguez ME, DomínguezReyes A. Breathing mode influence on craniofacial development and head posture. J Pediatr (Rio J). 2018;94(2):123-30. Disponible en: https://doi.org/10.1016/j.jped.2017.05.007

6. Leal R, Gomes M, Granville-García A, Goes P, de Menezes V. Impact of breathing patterns on the quality of life of 9- to 10-year-old schoolchildren. Am J Rhinol Allergy. 2016;30(5):147-52. Disponible en: https://doi.org/10.2500\%2Fajra.2016.30.4363

7. Sano M, Sano S, Oka N, Yoshino K, Kato T. Increased oxygen load in the prefrontal cortex from mouth breathing: a vector-based near-infrared spectroscopy study. Neuroreport. 2013;24(17):935-40. Disponible en: https://doi.org/10.1097/wnr.0000000000000008

8. ASG (GESIDA) of the SS of ID, Microbiology C, the National AIDS Plan. Executive summary of the GESIDA/National AIDS Plan Consensus Document on Antiretroviral Therapy in Adults Infected by the Human Immunodeficiency Virus (Updated January 2017). Enferm Infecc Microbiol Clin. 2018;36(7):435-45. Disponible en: https://doi.org/10.1016/j.eimc.2017.04.001

9. D'Onofrio L. Oral dysfunction as a cause of malocclusion. Orthod Craniofac Res. 2019;22(Suppl 1):43-8. Disponible en: https://doi.org/10.1111/ocr.12277

10. Thomé-Pacheco MC, Santos-Fiorott B, Silveiia-Finck N, Martins de Araújo MT. Craniofacial changes and symptoms of sleep-disordered breathing in healthy children. Dent Press J Orthod. 2015;20(3):80-7. Disponible en: https://doi.org/10.1590/2176-9451.20.3.080-087.oar

11. Franco L, Souki B, Cheib P, Abrão M, Pereira T, Becker H, et al. Are distinct etiologies of upper airway obstruction in mouth-breathing children associated with different cephalometric patterns? Int J Pediatr Otorhinolaryngol. 2015;79(2):223-8. Disponible en: https://doi.org/10.1016/j.ijporl.2014.12.013

12. Lee S-Y, Guilleminault C, Chiu H-Y, Sullivan S. Mouth breathing, "nasal disuse," and pediatric sleep-disordered breathing. Sleep Breath. 2015;19(4):1257-64. Disponible en: https://doi.org/10.1007/s11325-015-1154-6

13. Trevisan ME, Bellinaso JH, de Bitencourt-Pacheco A, Barrios-Augé L, Toniolo da Silva AM, Rodrigues-Correa E. Respiratory mode, nasal patency and palatine dimensions. CoDAS. 2015;27(2):201-6. Disponible en: https://doi.org/10.1590/2317-1782/20152014177

14. Milanesi J, Borin G, Rodrigues-Corrêa E, Toniolo da Silva AM, Bortoluzzi D, Souza J. Impact of the mouth breathing occurred during childhood in the adult age: biophotogrammetric postural analysis. Int J Pediatr Otorhinolaryngol. 2011;75(8):999-1004. Disponible en: https://doi.org/10.1016/j.ijporl.2011.04.018

15. de Moura Milanesi J, Berwig LC, Marquezan M, Schuch LH, de Moraes A, Toniolo da Silva AM, et al. Variables associated with mouth breathing diagnosis in children based on a multidisciplinary assessment. Codas. 2018;30(4):e20170071. Disponible en: https://doi.org/10.1590/2317-1782/20182017071

16. Abreau R, Rocha R, Lamounier J, Marques-Guerra AF. Etiology, clinical manifestations and concurrent findings in mouth breathing children. J Pediatr (Rio J). 
2008;84(6):529-35.

Disponible

en: https://doi.org/10.2223/jped.1844

17. Thomé-Pacheco MC, Ferreira-Casandrade C, PachecoTeixeira L, Finck N, Martins-de Araujo MT. Guidelines proposal for clinical recognition of mouth breathing children. Dent Press J Orthod. 2015;20(4):39-44. Disponible en: https://doi.org/10.1590/2176-9451.20.4.039-044.oar

18. Wieler W, Barros A, Barros L, Camargo S, Ignacio S, Maruo $\mathrm{H}$, et al. A combined protocol to aid diagnosis of breathing mode. Rev Clín Pesq Odontol. 2007;3(2):101-14. Disponible en:

https://periodicos.pucpr.br/index.php/oralresearch/article /view/23107

19. Diouf J, Diallo B, Diop-Ba K, Badiane A, Ngom P, Sonko O, et al. Relationships between the obstructive character of the tonsils and the type of ventilation and lip posture. Int Orthod. 2018;16(2):349-60. Disponible en: https://doi.org/10.1016/j.ortho.2018.03.007

20. Tiemi-Okuro R, Moreno-Morcillo A, Sakano E, SantosSchivinski CI, Gonclaves-Oliveira MA, Ribeiro J. Exercise capacity, respiratory mechanics and posture in mouth breathers. Braz j otorhinolaryngol. 2011;77(5):656-62. Disponible en: https://doi.org/10.1590/s180886942011000500020

21. Ikenaga $\mathrm{N}$, Yamaguchi $\mathrm{K}$, Daimon S. Effect of mouth breathing on masticatory muscle activity during chewing food. J Oral Rehabil. 2013;40(6):429-35. Disponible en: https://doi.org/10.1111/joor.12055

22. Kawamura Y. Physiology of Mastication. Front Oral Biol Basel, Karger. 1974;1:121-58.

23. Silvestrini-Biavati A, Salamone S, Silvestrini-Biavati F, Agostino P, Ugolini A. Anterior open-bite and sucking habits in Italian preschool children. Eur J Paediatr Dent. 2016;17(1):43-6. Disponible en: https://pubmed.ncbi.nlm.nih.gov/26949238/

24. Fiala M. Open bite and interdental sigmatism. Stomatol DDR. 1989;39(4):238-52. Disponible https://pubmed.ncbi.nlm.nih.gov/2623721/

25. Balaguer-García R. Valoración de un método de posturografía estática con pruebas dinámicas para evaluar funcionalmente pacientes vestibulares en edad laboral y su relación con el índice de discapacidad. Valencia; 2012. Universidad de Valencia. Disponible en: https://roderic.uv.es/bitstream/handle/10550/25455/TD \%20RAMON\%20BALAGUER\%20GARCIA.pdf?sequence=1

26. De-Moya P, Bertomeu B, Broseta V. Evaluacion y rehabilitacion del equilibrio mediante posturografia. Rehabilitación. 2005;39(6):315-23. Disponible en: https://doi.org/10.1016/S0048-7120(05)74365-6

27. Huo F. Limits of stability and postural sway in young and older people; 1999. Ontario: University of Queens.
Disponible en: https://www.collectionscanada.gc.ca/obj/s4/f2/dsk1/tape 9/PQDD_0005/MQ42635.pdf

28. Basmajian JV. Electromyography--dynamic gross anatomy: a review. Am J Anat. 1980;159(3):245-60. Disponible en: https://doi.org/10.1002/aja.1001590302

29. Ferrario V, Sforza C, Miani A, D’Addona A, Barbini E. Electromyographic activity of human masticatory muscles in normal young people. Statistical evaluation of reference values for clinical applications. J Oral Rehabil. 1993;20(3):271-80. Disponible en: https://doi.org/10.1111/j.1365-2842.1993.tb01609.x

30. Ortiz F, Rincón R, Mendoza J. Texto de medicina fisica y rehabilitacion. Bogota: Editorial Menual Moderno; 2016.

31. Ono $\mathrm{T}$, Ishiwata $\mathrm{Y}$, Kuroda $\mathrm{T}$. Inhibition of masseteric electromyographic activity during oral respiration. American Journal of Orthodontics and Dentofacial Orthopedics. 1998;113(5):518-25. Disponible en: http://www.duttondds.com/wpcontent/uploads/2014/02/Ono-1998-AJODO-113-51825.pdf

32. Hsu H-Y, Yamaguchi K. Decreased chewing activity during mouth breathing. J Oral Rehabil. 2012;39(8):559-67. Disponible en: https://doi.org/10.1111/j.13652842.2012.02306.x

33. Cuccia A, Lotti M, Caradonna D. Oral Breathing and head posture. Angle Orthod. 2008;78(1):77-82. Disponible en: https://doi.org/10.2319/011507-18.1

34. da Silveira W, de Queiroz F, Silva F, Silveira SL. Postural alterations and pulmonary function of mouth breathing children. Braz J Otorhinolaryngol. 2010;76(6):683-6. Disponible en: https://doi.org/10.1590/S180886942010000600002

35. Neiva P, Kirkwood R, Mendes P, Zabjek K, Becker H, Mathur S. Postural disorders in mouth breathing children: a systematic review. Braz J Phys Ther. 2018;22(1):7-19. Disponible en: https://doi.org/10.1016/j.bjpt.2017.06.011

36. Yi L, Jardim J, Inoue D, PIgnatari S. The relationship between excursion of the diaphragm and curvatures of the spinal column in mouth breathing children. J Pediatr (Rio J). 2008;84(2):171-7. Disponible en: https://doi.org/10.2223/jped.1771

37. Martínez-Nova A, Sanchez-Rodríguez, R. Cuevas-García J, Sanchez-Barrado E. Estudio baropodométrico de los valores de presión plantar en pies no patológicos. Rehabilitacion. 2007;41(4):155-60. Disponible en: https://medes.com/publication/36012

38. Tafner BN. Analise estabilometrica da influencia da oclusao na postura ortostatica. Blumenau: Universidade Regional de Blumenau; 2010. Disponible en: https://bu.furb.br//docs/mo/2011/345311_1_1.pdf 